\title{
AN ADAPTIVE FEEDFORWARD CONTROL METHOD FOR UNDER-ACTUATED BIPEDAL WALKING ON THE COMPLIANT GROUND
}

\author{
Yang Wang, ${ }^{*}$ Jiatao Ding, ${ }^{*}$ and Xiaohui Xiao*
}

\begin{abstract}
Motivated by the potential use of humanoid robot in real environment, an adaptive feedforward control strategy is developed to stabilize the underactuated bipedal walking on the compliant ground. First, the robot-ground coupling dynamic system is modelled as a rigid kinematical chain coupled with a spring-damper system. Then by observing the human's gait, we find the walking speed has a direct effect upon the walking stability. In consideration of the highly complicated impact of real road surface on direct walking speed control, through analysis on laws governing the robot's walking speed and the centre-of-mass (CM) motion, we establish a parameterized equivalent rod-ground coupling model based on the robot's actual state, and identify the mapping relation between its walking speed and the CM forward moving distance of a full walking cycle $\left(x_{f}\right)$ adaptively. Finally, the robot's walking is stabilized through a feedforward control over $x_{f}$. The availability and adaptability of this method were validated through simulations: specific to one initial gait and three compliant conditions with different damping parameters, the walking was stabilized and the rate of stable convergence improved; specific to three initial gaits and three compliant conditions with stochastic varying damping parameters, the walking was still stabilized and its performance also improved.
\end{abstract}

\section{Key Words}

Underactuated, bipedal walking, compliant ground, adaptive feedforward control

\section{Introduction}

A three-link planar bipedal robot walking on a level ground is studied in this paper. The walking process is characterized by the underactuation at the robot-ground interface [1]. Because of the high manoeuvrability and low cost of system resource, underactuated bipedal walking robot has been studied prevalently [2], [3]. Some famous bipedal robots, such as RABBIT [4], ATRIAS [5], ATLAS [6], MABEL [7], and AMBER [8], were published. On the other

* School of Power and Mechanical Engineering, Wuhan University, Hubei Province, 430072, P.R. China; e-mail: wangyang. hf1987@whu.edu.cn,jtdingx@163.com,xhxiao@whu.edu.cn Corresponding author: Xiaohui Xiao

Recommended by Prof. James Yang

(DOI: 10.2316/Journal.206.2017.1.206-4779) hand, in virtue of high adaptability and acceptability in human society, the potential application of humanoid robot is universal, ranging from housework to disaster relief [9]. As the result, the rigid robot-ground contact model would be not applicable for all road surface materials, especially when the robot is carrying heavy equipment and marching at a quick pace [10], [11]. In this paper, the ground is compliant and modelled as the spring-damper system, furthermore, an adaptive feedforward control strategy is proposed to stabilize the underactuated bipedal walking on the compliant ground.

The compliant robot-ground contact model differs from the rigid in the following three aspects. In the perturbation description aspect, because of the difficulty in detecting the exact ground compliance by non-contact technology, the exact effect of the ground on the walking stability is unpredictable before the robot actually stepping on it. In the robot's walking state aspect, because the motion of the robot-ground contact point $(\mathrm{CP})$ is coupled with the ground deformation in global coordinates, the robot is essentially underactuated throughout a full walking cycle. In the system characteristic aspect, the whole walking process is continuous and the robot's joints velocity cannot jump.

In the literature, the effect of ground compliance on the underactuated bipedal walking was first explored in the experiments with the robot RABBIT [12], [13]. In the numerical experiment, when RABBIT was walking on the compliant surface, the walking speed was lower than in the rigid case [12]. Similarly, in the physical experiment, when RABBIT was stepping on a level steel plate, it was also slower than on the concrete floor significantly [13]. In these documents, these phenomena were all attributed to the increased energy dissipation during compliant robotground impact [12], [13]. Recently, in the experiments with MABEL, as the robot's actual walking speed was always faster than the predicted, a factor had to be used to scale the theoretical impact model for matching the simulation to the experimental results [14]. From the above, the effect of ground compliance on the robot's walking performance actually exists and the theoretical rigid robot-ground contact model is inapplicable to realize a stable and efficient underactuated bipedal walking in real environment. 
The control strategies for stabilizing the underactuated bipedal walking in complex ground environment could be classified into two groups. The first is focused on the stable walking on uneven terrain [15]-[20]. In these studies, the robot-ground contact was rigid and the disturbance of the ground on the walking stability was describable [21]. However, as the two pre-conditions are inapplicable when the ground is compliant, these control strategies are also inapplicable to stabilize the walking on compliant ground. The second group is based on the relationship between the robot's walking stability and its speed. Specifically speaking, in [22], based on a set of preplanned gaits with different walking speeds, a switched control strategy was developed for the robot prototype ERNIE to realize a stable walking on a treadmill with varying speed; in [23] and [24], by applying the artificial neural network method, the mapping relation between the robot prototype Runbot's walking speed and each joint state was identified, and then the walking stability was improved by regulating the walking speed through the control of each joint motion; in [25], by analysing the effect of robot prototype Meta's three parameters, the amount of ankle push-off, upper body pitch, and step length, on the walking speed, the walking stability was also improved by the control of walking speed. Although the stabilization through the control of robot's walking speed has been realized in some physical experiments, these methods have two drawbacks. The first is low level. As these control strategies are focused on the relationship between the robot's walking speed and the single or multiple joints motions, when the bipedal robot's degrees of freedom is high, the small modelling or tracking error of each joint should result in a dramatic deviation of the robot's walking performance from the desired. The second is low adaptability. In spite of the independence from the theoretical robot-ground contact model, these methods are still based on the pre-sampled data which is further determined by the conditions of modelling procedures, such as the experiment ground condition, the specific prototype robot's mechanism, the preplanned gait, and even the data sampling method. As a result, these methods are essentially non-general for all underactuated bipedal walking system. Based on the above, in this paper, an adaptive feedforward control strategy which is focused on the control of robot's CM motion to regulate the walking speed and further stabilize the whole walking process on compliant ground is proposed.

Specifically speaking, this adaptive feedforward control strategy is developed in consideration of the following three aspects: (1) for a robot with multiple degree-of-freedom, the trajectory of the centre-of-mass (CM) is determined by the trajectories of its joints, meanwhile, the horizontal velocity of the $\mathrm{CM}$ is always positively related to the robot's walking speed; (2) when a man is walking on a level floor, the speed will be increased when his body leans forward and decreased when his body leans backward; (3) for the cyclic walking, the whole process will be stabilized if its walking speed can always converge to a desired value which has been proved available for realizing at least one cycle walking. With this method, the walking stabilization control is equated into the regulation of robot's walking speed and further realized through a single-input-singleoutput (SISO) control of its CM motion.

This paper is organized as follows. First, the robotground coupling dynamic system is modelled as a rigid planar kinematical chain in coupled with a spring-damper system. Second, inspired by the human's gait and based on the robot's walking state, a low dimensional parameterized equivalent model for identifying the mapping relation between the robot's CM trajectory and horizontal velocity on the compliant ground is established and the desired gait in the next cycles is figured out through a simple inverse calculation upon it. Finally, to validate the availability and the adaptability of this control strategy, various initial gaits and ground damping parameters are considered in the simulation experiments.

\section{Model of Underactuated Bipedal Walking on Compliant Ground}

\subsection{Rigid Robot Configuration}

The planar robot is composed of a hips $\left(q_{3}\right)$, two knees $\left(q_{2}, q_{4}\right)$, two thighs $\left(l_{2}, l_{3}\right)$, two calves $\left(l_{1}, l_{4}\right)$, but no ankle. The three joints $\left(q_{2}, q_{3}, q_{4}\right)$ are controllable. The structure description and the definition of the generalized coordinates $q=\left[q_{1}, q_{2}, q_{3}, q_{4}, x_{s t}, y_{s t}\right]^{\prime}$ are indicated in Fig. 1(a). In particular, the masses are distributed, and the position angles of joints are computed counter-clockwise except $q_{1}$.

\subsection{Compliant Ground Equivalence}

The compliant ground is constructed with a group of independent compliant structure, as shown in Fig. 1(b), where the stiffness of the elastic spring is denoted by $k$ and the damping of viscous damper denoted by $c$. Each surface fragment is connected to the rigid ground foundation through a pair of spring-damper units in horizontal and vertical direction, respectively. Three constraints for the compliant ground model are that [26]-[28]:

1. The surface fragment is rigid, zero-thickness, and massless. There is no energy consumption for the inertia of ground surface during the full walking cycle.

2. The ground surface is extremely rough. The energy consumption for the friction at robot-ground interface is equated to a part of the viscous and elastic force generated by the ground compliance.

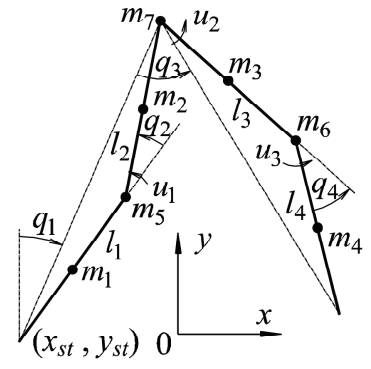

(a)

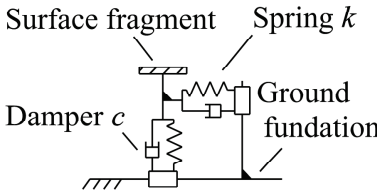

(b)
Figure 1. Models of rigid robot and compliant ground structure. 


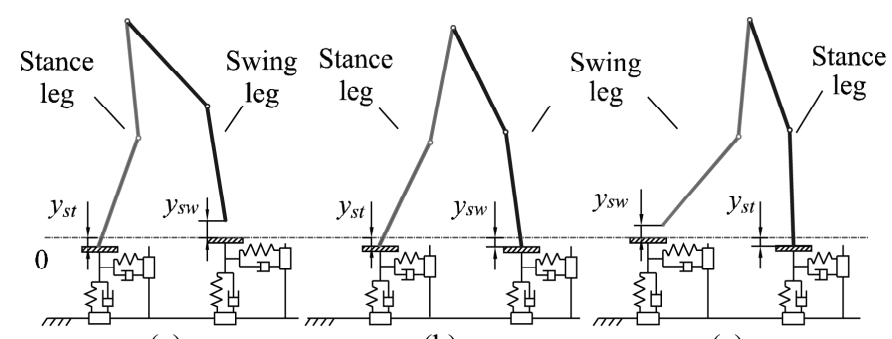

(a)

(b)

(c)

Figure 2. Walking on compliant ground. (a) SSP; (b) DSP; and (c) SSP.

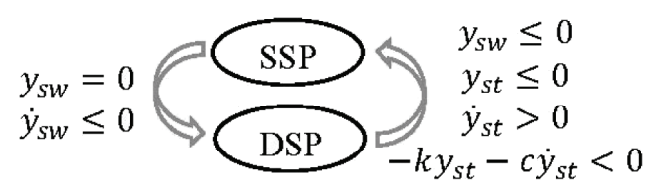

Figure 3. Switch between SSP and DSP.

3. The spring-damper unit is only deformed axially. The properties of both units in a compliant structure are identical.

4. The global coordinates are fixed to the ground foundation. The initial vertical position of every surface fragment is on the zero level.

\subsection{Definition of a Full Walking Cycle}

The definition of a full walking cycle under compliant robot-ground assumption is described in Fig. 2 and follows four constraints:

1. The walking cycle only takes place in the sagittal plane.

2. A full walking cycle consists of two non-transient successive phases, a single support phase (SSP) and a double support phase (DSP) ("single" and "double" is corresponding to the situation that only one leg and both legs are standing on the ground, respectively).

3. During the SSP, the label of each leg is determined by its actual role. Specifically speaking, during a SSP, the leg standing on the ground is labelled with "stance" and the other labelled with "swing". While during the DSP, the label of each leg is determined by its role in the previous SSP. It means that, during a DSP, the leg labelled with "swing" in the previous SSP is still labelled with "swing", and the leg labelled with "stance" in the previous SSP still labelled with "stance".

4. The DSP starts when the swing leg strikes the ground and terminates when the stance leg lifts apart from the ground. The switch conditions between a SSP and a DSP are shown in Fig. 3.

Based on the Poisson's hypothesis, the contact between a foot ("foot" indicates the point on the bottom of a calf) and the ground beneath it is separated into a compression phase followed by a restitution phase [29]. The relationship between the robot-ground contact phases and the footground contact phases is described in Fig. 4.

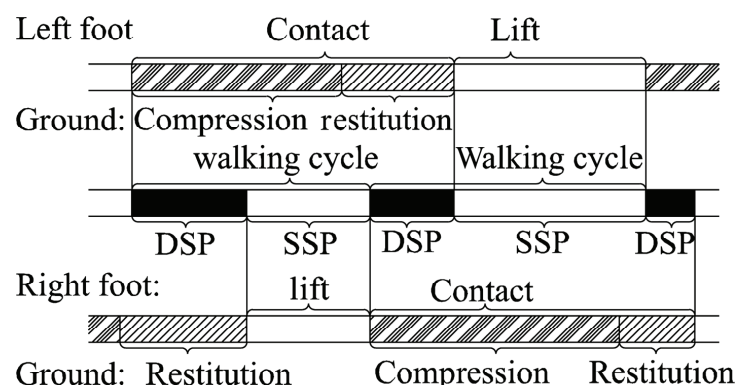

Figure 4. Schedule of walking cycle.

\subsection{Coupling Dynamics Model During SSP}

During a SSP, the stance leg is acting as a pivot, and the robot-ground system is modelled as a rigid planar kinematic chain in one-point contact with a stiffness-damping system, as shown in Fig. 2(a) and (c). By using the method of Lagrange, the robot-ground coupling dynamical system during a SSP is described with a standard second-order equation of motion:

$$
M(q) \ddot{q}+H(q, \dot{q})=B_{1} F_{s t}(q, \dot{q})+B_{2} u
$$

with

$$
\begin{aligned}
F_{s t}(q, \dot{q}) & =\left[\begin{array}{c}
-c \dot{x}_{s t}-k\left(x_{s t}-x_{s t 0}\right) \\
-c \dot{y}_{s t}-k y_{s t}
\end{array}\right] \\
B_{1} & =\left[\begin{array}{llll}
0 & \cdots & 1 & 0 \\
0 & \cdots & 0 & 1
\end{array}\right]_{2 \times 6}^{\prime} \\
B_{2} & =\left[\begin{array}{ccc}
0 & \cdots & 0_{1 \times 2} \\
\vdots & I_{3} & \vdots \\
0 & \cdots & 0_{2 \times 2}
\end{array}\right]_{6 \times 6}^{\prime}
\end{aligned}
$$

where $M(q)$ is the $(6 \times 6)$ positive-definite mass-inertia matrix, $H(q, \dot{q})$ is the $(6 \times 1)$ vector of Coriolis and gravity terms, $u$ is the $(3 \times 1)$ vector of input torques, $x_{s t 0}$ is the initial horizontal position of the stance foot when it strikes the ground surface in the previous DSP, and $F_{s t}$ is the reaction forces from the ground acting on the stance foot. During this phase, $q_{2}, q_{3}$, and $q_{4}$ are controlled with torques $u_{1}, u_{2}$, and $u_{3}$, respectively, but $q_{1}, x_{s t}$ and $y_{s t}$ are underactuated. Thus, the whole robot is underactuated. The state-space form for this second-order system is written as:

$$
\begin{aligned}
\dot{s} & =\frac{d}{d t}\left[\begin{array}{c}
q \\
\dot{q}
\end{array}\right]=\left[\begin{array}{c}
\dot{q} \\
M^{-1}(q)\left[-H(q, \dot{q})+B_{1} F_{s t}(q, \dot{q})+B_{2} u\right]
\end{array}\right] \\
& =h(s)+f_{s t}(s)+b(s) u
\end{aligned}
$$




\subsection{Coupling Dynamics Model During DSP}

The DSP phase is non-transient. Compared with (1), another force from ground will work on the swing leg. And then, the robot-ground system is modelled as a rigid planar kinematic chain in two-point contact with a stiffnessdamping system, as shown in Fig. 2(b). Still by using the method of Lagrange, the robot-ground coupling dynamical system during a DSP is also described with a second-order equation of motion:

$$
\begin{gathered}
M(q) \ddot{q}+H(q, \dot{q})=B_{1} F_{s t}(q, \dot{q})+E_{s w}^{\prime} F_{s w}(q, \dot{q})+B_{2} u \\
F_{s w}(q, \dot{q})=\left[\begin{array}{c}
-c \dot{x}_{s w}-k\left(x_{s w}-x_{s w 0}\right) \\
-c \dot{y}_{s w}-k y_{s w}
\end{array}\right]
\end{gathered}
$$

where $E_{s w}$ is a $(2 \times 6)$ Jacobian matrix, $x_{s w}$ and $y_{s w}$ are the horizontal and vertical positions of swing foot in global coordinates, respectively, $x_{s w 0}$ is the initial horizontal position of the swing foot when it strikes the ground surface, and $F_{s w}$ is the reaction force from the ground acting on the swing foot. During this phase, as $x_{s t}$ and $y_{s t}$ are both underactuated, the whole robot is still underactuated. The state-space form for this second-order system is written as:

$$
\begin{aligned}
\dot{s} & =\frac{d}{d t}\left[\begin{array}{l}
q \\
\dot{q}
\end{array}\right] \\
& =\left[\begin{array}{c}
\dot{q} \\
M^{-1}(q)\left[-H(q, \dot{q})+B_{1} F_{s t}(q, \dot{q})+B_{2} u+E_{s w}(q) F_{s w}(q, \dot{q})\right]
\end{array}\right] \\
& =h(s)+f_{s t}(s)+b(s) u+f_{s w}(s)
\end{aligned}
$$

\subsection{Overall Model}

Combining these two models, the overall model for the underactuated bipedal walking on the compliant ground is expressed as:

$$
\begin{cases}\dot{s}=h(s)+f_{s t}(s)+b(s) u & y_{s w}>0 \\ \dot{s}=h(s)+f_{s t}(s)+b(s) u+f_{s w}(s) & y_{s w} \leq 0\end{cases}
$$

When the swing foot strikes the ground, the robotground coupling dynamical system is switched into the DSP and described with the second equation of (5). When the stance leg lifts from the ground, the roles of the both legs are exchanged with each other, the robot-ground coupling dynamical system is switched into the SSP and described with the first equation of (5), simultaneously. The final state of each phase is the initial state of the following one.

\subsection{Simulation of Uncontrolled Walking on the Compliant Ground}

\subsubsection{Gait Plan Under Rigid Contact Assumption}

To initialize an underactuated bipedal walking on compliant ground, an asymptotically stable gait acting as the initial robot's state must be preplanned under the rigid robot-ground contact assumption.

Following the design procedure based on Poincaré return map method applied in [30] and [31], in this paper, the trajectories of the three actuated joints are also expressed as the functions of the variable $q_{1}$ (the underactuated joint, shown in Fig. 1) and described with the three-degree Bézier polynomials. With the initial and final robot's states, denoted by $\left[q_{i}, \dot{q}_{i}\right]^{\prime}$ and $\left[q_{f}, \dot{q}_{f}\right]^{\prime}$ respectively, the trajectory of each actuated joint, $q_{a}$ (the subscript " $a$ " denotes the actuated joint, $a=2,3$, and 4 ), is obtained. Set

$$
\begin{gathered}
q_{i}=\left[q_{1 i}, q_{2 i}, q_{3 i}, q_{4 i}\right]^{\prime} \quad \dot{q}_{i}=\left[\dot{q}_{1 i}, \dot{q}_{2 i}, \dot{q}_{3 i}, \dot{q}_{4 i}\right]^{\prime} \\
q_{f}=\left[q_{1 f}, q_{2 f}, q_{3 f}, q_{4 f}\right]^{\prime} \quad q_{f}=\left[\dot{q}_{1 f}, \dot{q}_{2 f}, \dot{q}_{3 f}, \dot{q}_{4 f}\right]^{\prime}
\end{gathered}
$$

then

$$
q_{a}\left(q_{1}\right)=\sum_{k=0}^{3} \alpha_{k} \frac{3 !}{k !(3-k) !} s^{k}(1-s)^{3-k}
$$

where

$$
s=\frac{q_{1}-q_{1 i}}{q_{1 f}-q_{1 i}}
$$

The coefficients of the Bézier polynomial are obtained through:

$$
\begin{aligned}
& \alpha_{0}=\left(q_{i}\right)_{a} \\
& \alpha_{0}=\left(q_{i}\right)_{a}+\frac{q_{1 f}-q_{1 i}}{3} \frac{\left(\dot{q}_{i}\right)_{a}}{\dot{q}_{1 i}} \\
& \alpha_{2}=\left(q_{f}\right)_{a}-\frac{q_{1 f}-q_{1 i}}{3} \frac{\left(\dot{q}_{f}\right)_{a}}{\dot{q}_{1 f}} \\
& \alpha_{3}=\left(q_{f}\right)_{a}
\end{aligned}
$$

\subsubsection{Simulation of Uncontrolled Walking on Compli- ant Ground}

The walking process on a compliant ground starts with a SSP (at the very beginning, $x_{s t}=0, \dot{x}_{s t}=0, y_{s t}=0$, and $\left.\dot{y}_{s t}=0\right)$ and the initial robot state is assigned with $\left[q_{i}, \dot{q}_{i}\right]^{\prime}$.

During a SSP, the desired trajectories of actuated joints are expressed as (6) and generated online at the beginning of this phase. In the first cycle, the initial and final state for the desired trajectories generation is assigned with $\left[q_{i}, \dot{q}_{i}\right]^{\prime}$ and $\left[q_{f}, \dot{q}_{f}\right]^{\prime}$, respectively. After the first cycle, the initial state for the trajectories generation is updated with the actual robot's state at the end of the previous DSP, but the final state for the generation still assigned with $\left[q_{f}, \dot{q}_{f}\right]^{\prime}$. 
For analysing the effect of ground compliance, the actuated joints are non-friction and the torques applied on the joints, $u_{1}, u_{2}$, and $u_{3}$, are all set to zero during the DSP. Then, (5) is simplified into:

$$
\begin{cases}\dot{s}=h(s)+f_{s t}(s)+b(s) u & y_{s w}>0 \\ \dot{s}=h(s)+f_{s t}(s)+f_{s w}(s) & y_{s w} \leq 0\end{cases}
$$

In the technical perspective, with this setting, the over-stiffness problem when calculating the robot-ground coupling motion during a DSP numerically is avoided [13]-[27]. Furthermore, as the robot's actual state during this phase is only determined by its initial state at the beginning of a DSP and the effect of gravity and ground compliance, this situation is the worst case for stabilization control. As the result, if the controlled walking system can be stabilized even under this situation, when these actuated joint controllable, the controlled robot must walking stably on compliant ground.

\section{Adaptive Feedforward Control Strategy}

It must be noted that to discriminate our work from the study about walking on uneven terrain, the stiffness of the compliant ground structure is set to large, for which the ground deformation is so small that the effect of unevenness is limited.

\subsection{Objective of Stabilization Control}

As the underactuated bipedal walking system is a multivariable cyclic dynamical system, to realize a SISO control, the controlled input and output must be selected elaborately.

In consideration of three perspectives: the cyclic dynamical system characteristic, physical experiment realization, and model simplification, the robot's CM horizontal velocity at the end of a $\operatorname{SSP}\left(u_{f}\right.$, as shown in Fig. 5) is selected as the controlled output of the SISO system. First, in the cyclic dynamical system characteristic perspective, $u_{f}$ describes the total variation of the CM horizontal velocity through a full walking cycle. Second, in the physical experiment realization perspective, the time for sensing the value of $u_{f}$ can be detected easily just by identifying the sharp increment of the pressure on the lower surface of swing foot. Last but most important, in the model simplification perspective, because of that, with selecting $u_{f}$ as the controlled output, during a DSP and the following SSP, the robot's CM position can be described with the coordinates relative to the $\mathrm{CP}$ between the ground and the leg labelled with "swing" in the DSP, as shown in Fig. 2(b) and (c), the high-dimension original system can be equated into a SISO system.

Meanwhile, inspired by the characteristic of human's gait aforementioned (when the body leans forward, the walking speed increases, and when the body leans back, the walking speed decreases automatically), when the robot's step length is a constant, the CM forward moving distance

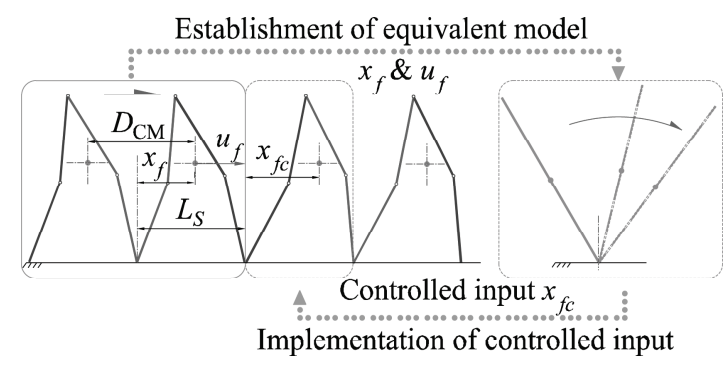

Figure 5. Schematic diagram of adaptive feedforward control strategy $\left(D_{C M}\right.$ is the distance of robot's CM moving through a full walking cycle; walking speed $:=D_{\mathrm{CM}} /$ period of a full walking cycle).

of a full walking cycle $\left(x_{f}\right.$, as shown in Fig. 5) is selected as the controlled input.

Based on the above, a SISO control system, in which $x_{f}$ is the controlled input and $u_{f}$ the controlled output, is constructed. By identifying the mapping relation between $u_{f}$ and $x_{f}$ under the effect of the compliant ground and figuring out the robot's gait corresponding to this ground effect, the control of robot's walking speed is realized and the whole walking process will be stabilized.

In this paper, to identify the mapping relation between $u_{f}$ and $x_{f}$ under the effect of ground compliance adaptively, an equivalent rod-ground coupling dynamical model only based on the robot actual state is established, and then, the controlled input can be derived through a simple inverse operation upon it. The schematic diagram of the adaptive feedforward control strategy is described as Fig. 5. To implement the control strategy on the real robot system, the mapping relationship between the controlled $x_{f}, x_{f c}$, and the controlled final robot configuration of SSP, $q_{f c}$, must be modelled, which is detailed in Appendix 1.

\subsection{Model Equivalence}

Corresponding to the both phases of a full walking cycle, two sub-models are established separately. Furthermore, considering the controlled output $u_{f}$ being the output of the SISO control system, the two sub-models are constructed in the sequence, the impact submodel first and the swing following, as shown in Fig. 6. According to it, two constraints must be satisfied for establishing the equivalent rod-ground coupling dynamical model:

1. The initial rod's CM horizontal velocity of the impact submodel must be identical with the initial robot's CM horizontal velocity during the DSP; the final rod's CM horizontal velocity of the swing submodel must be identical with the final robot's CM horizontal velocity during the following SSP.

2. The initial rod's CM horizontal position relative to the rod-ground CP of the impact submodel must be identical with the initial robot's CM horizontal position relative to the robot-ground $\mathrm{CP}$ during the DSP. The final rod's CM horizontal position relative to the rodground $\mathrm{CP}$ of the swing submodel must be identical with the final robot's CM horizontal position relative to the robot-ground CP during the following SSP. 


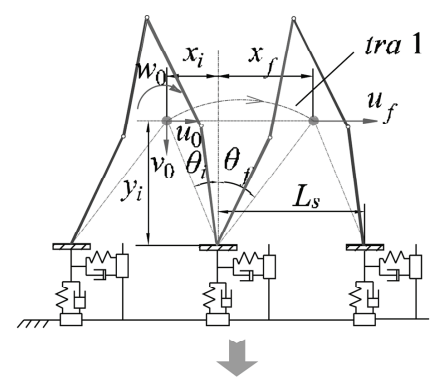

(a)

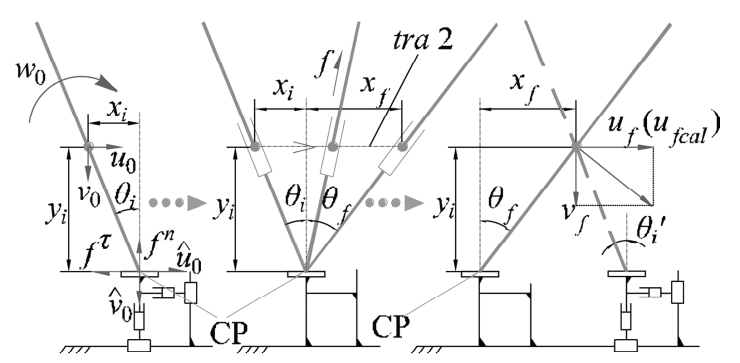

(b)

(c)

(d)

Figure 6. Equivalent motion system. (a) Original model; (b) impact sub-model; (c) swing sub-model; and (d) final state of overall model. (tra1: the real trajectory of the robot's CM; tra2: the designed trajectory of invert pendulum.)

\subsubsection{Impact Submodel}

Corresponding to the DSP, the equivalent impact submodel is established. During a DSP, the robot's both legs are standing on the ground and the force for supporting robot's CM motion is transferred from the stance leg to the swing leg.

First, as the duration of DSP is short and the variation of robot configuration very small, the rod-ground impact is considered as a time-dependent process, where only changes in momenta occur without changes in configuration. As a result, in the equivalent impact submodel, the rod is rigid and the effect of compliant ground on the rod is only the viscous force related to the ground damping parameter. The rigid-rod-damped-ground model is shown in Fig. 6(b).

Second, according to the switch condition shown in Figs. 3 and 4, the DSP terminates when the stance leg lifts apart from the ground surface. Correspondingly, in the equivalent model, an indicator is also needed for measuring the duration of a DSP. Thus, in this paper, by considering the actual effect of the ground compliance on the robot's CM motion during a DSP, a coefficient used to measure the decrement of the tangential contact velocity at the rod-ground CP is introduced to work as the indicator.

Compared with the researches about the rigid body non-collinear collision in literature [10] and [28], the ground in this paper is compliant in both normal and tangential direction. This situation has not been discussed further before.

During the impact phase, the rod's CM moves towards the ground surface with speed $v$ and tangential to the surface with speed $u$. Meanwhile, the body rotates with angular speed $w$. At the $\mathrm{CP}$, there is a normal component of relative velocity $\hat{v}=v+x w$ and a tangential component $\hat{u}=u-y w$, where $(x, y)$ is the position of the rod's CM relative to the CP. Suppose the CM of the rigid rod has an initial velocity $v=\left(v_{0}, u_{0}\right)$ and initial angular speed $w_{0}$. Meanwhile, the initial position of the rod's CM relative to the $\mathrm{CP}$ is $r=\left(x_{i}, y_{i}\right)$. Hence there is an initial contact velocity $\left(\hat{v}_{0}, \hat{u}_{0}\right)=\left(v_{0}+x_{i} w_{0}, u_{0}-y_{i} w_{0}\right)$. Impulsemomentum relations for the rod motion yield:

$$
\left\{\begin{array}{l}
v(t)-v_{0}=-p^{n} / m \\
u(t)-u_{0}=-p^{\tau} / m \\
w(t)-w_{0}=\left(x_{i} p^{n}+y_{i} p^{\tau}\right) / \rho^{2} m
\end{array}\right.
$$

where $t$ is the time variable, $m$ is the total mass of the robot, $p^{\tau}$ and $p^{n}$ denote the tangential and normal reaction impulses acting at the $\mathrm{CP}$, respectively, and $\rho\left(\rho:=\sqrt{\left(x_{i}^{2}+y_{i}^{2}\right) / 3}\right)$ is the gyration radius of the rod.

The final state of the rod's CM is deduced in two steps. First, assume there is no effect in normal direction. The deceleration of the tangential contact velocity at $\mathrm{CP}$ is given by:

$$
\hat{u}=\hat{u}_{0}-p^{\tau} / \frac{\rho^{2} m}{\rho^{2}+y_{i}^{2}-x_{i} y_{i} p^{n} / p^{\tau}}
$$

Let $t_{1}$ denote the duration of impact phase and $\lambda$ the dissipation ratio of the tangential contact velocity through this phase. As the decrement of the tangential contact velocity is proportional to $p^{\tau}$, when $\hat{u}\left(t_{1}\right)=(1-\lambda) \hat{u}_{0}$ and $p^{n}=0$, with (9) and (10) yields:

$$
\begin{gathered}
p^{\tau}\left(t_{1}\right)=\lambda \frac{\rho^{2} m}{\rho^{2}+y_{i}^{2}} \hat{u}_{0} \\
\tilde{w}\left(t_{1}\right)=w_{0}+\lambda y_{i} \frac{1}{\rho^{2}+y_{i}^{2}} \hat{u}_{0} \\
\tilde{\hat{v}}\left(t_{1}\right)=v_{0}+\tilde{w}\left(t_{1}\right) x_{i}
\end{gathered}
$$

With the final condition (11) and the theorem of impulse, $t_{1}$ is calculated through solving an ordinary differential equation in the tangential direction:

$$
\begin{gathered}
\dot{f}^{\tau}=-c \frac{f^{\tau}}{m} \\
p^{\tau}\left(t_{1}\right)=-\int_{0}^{t_{1}} f^{\tau} d t
\end{gathered}
$$

Then, $t_{1}$ is given as:

$$
t_{1}=\ln \left[1-\frac{\rho^{2} m \lambda}{c\left(\rho^{2}+y_{i}^{2}\right)}\right]
$$

By applying Tayler's theorem, $t_{1}$ is linearized as:

$$
t_{1}=\frac{\rho^{2} m \lambda}{c\left(\rho^{2}+y_{i}^{2}\right)}
$$


Second, examine the variation of $\hat{v}(t)$. If a normal walking cycle will be realized, $w(t)$ must be non-negative during this phase. Thus, it must be tenable that $\hat{v}_{0} \leq \hat{v}\left(t_{1}\right) \leq \tilde{\hat{v}}\left(t_{1}\right)$. With the initial value $v_{0}$ and (13), by applying the mean value theorem, the normal reaction impulse $p^{n}$ during the impact phase is given approximately:

$$
p^{n} \approx \frac{t_{1}}{2}\left[c \hat{v}_{0}+c \tilde{\hat{v}}\left(t_{1}\right)\right]
$$

Then, with (7), (9), (12), and (18), the final vertical velocity and angular speed is modified as:

$$
\left\{\begin{array}{l}
u\left(t_{1}\right)=u_{0}-\frac{\rho^{2} m \lambda}{\rho^{2}+y_{i}^{2}} \hat{u}_{0} / m \\
v\left(t_{1}\right)=v_{0}-\frac{\rho^{2} m \lambda}{\rho^{2}+y_{i}^{2}}\left(\hat{v}_{0}+\frac{\tilde{v}\left(t_{1}\right)}{2}\right) / m \\
w\left(t_{1}\right)=w_{0}+\frac{\lambda y_{i}}{\rho^{2}+y_{i}^{2}} \hat{u}_{0}-\frac{\lambda x_{i}}{\rho^{2}+y_{i}^{2}}\left(\hat{v}_{0}+\frac{\tilde{w}\left(t_{1}\right) x_{i}}{2}\right)
\end{array}\right.
$$

\subsubsection{Swing Submodel}

Corresponding to the SSP, the equivalent swing submodel is established. During a SSP, the kinetic energy variation of the robot's CM is only related to the trajectory of itself during the SSP. Accordingly, the rigid rod is modified as a length-variable-inverted-pendulum. The design of the inverted pendulum's CM trajectory follows four aspects:

1. It is inefficient to fit the actual trajectory of the robot's CM during a full cycle in practice.

2. For periodic stable walking, the vertical positions of robot's CM at the beginning and the end of a full walking cycle are on the same level, as shown in Fig. 6(a).

3. In common sense, for a natural human-like gait, the vertical component of the CM trajectory is very small compared to the horizontal component.

4. Covering the three points above, and the tiny difference in the vertical component of the CM trajectory has little effect on real total work applied on the pendulum. Thus, for simplicity, a straight-line trajectory is designed as the inverted pendulum's CM trajectory for the swing submodel, as shown in Fig. 6(c). As the ground deformation is limited by the large ground stiffness parameter, the compliant ground is simplified to be rigid. And then, the total kinetic energy variation of the invert pendulum's CM is equal to the work applied on the pendulum axially which is further determined by the initial and final position of the inverted pendulum's CM. Furthermore, to diminish the error of the total kinetic energy variation between the robot $\mathrm{CM}$ and the invert pendulum model, another coefficient $\lambda^{\prime}$ is introduced. The axial work applied on the pendulum is deduced by:

$$
\begin{aligned}
\Delta W_{\text {pend }} & =\int_{\theta_{i}}^{\theta_{f}} f d r=\int_{\theta_{i}}^{\theta_{f}} \frac{m g}{\cos (\theta)} d\left(\frac{y_{i}}{\cos (\theta)}\right) \\
& =\frac{m g L_{S}\left(L_{S}-2 x_{i}\right)}{2 y_{i}}
\end{aligned}
$$

where $L_{S}$ is the designed step length, $\theta_{i}$ and $\theta_{f}$ denote the initial and final angles of a swing phase, respectively, as shown in Fig. 6. Then, the actual variation of kinetic energy through the swing phase is:

$$
\Delta W=\lambda^{\prime} \Delta W_{\text {pend }}
$$

\subsubsection{Overall Equivalent System}

First, to construct the overall equivalent model begins with the impact model and end with the swing model, subject to the conservation of kinetic energy, with (19)-(21), and (23), the kinetic energy of the rod' CM at the end of a full cycle is calculated as:

$$
T_{\text {frod }}=\frac{m}{2}\left[v\left(t_{1}\right)^{2}+u\left(t_{1}\right)^{2}+\rho^{2} w\left(t_{1}\right)^{2}\right]+\Delta W
$$

Second, duo to the significant difference in structure between the original robot-ground model and the equivalent rod-ground model, the assignment of $w_{0}$ and the calculation of rod's CM horizontal velocity at the end of a swing phase, $u_{f c a l}$, must be further discussed. In the impact model, as the robot is actual a multi-rigid-body system and the rod is only a single rigid body, subject to the constraints for the equivalent model establishment, the value of $w_{0}$ is actually unsolvable. On the other hand, in the swing submodel, according to the designed trajectory, the total rod's kinetic energy variation will only contributes to its CM horizontal velocity changing, which is not in accord with the reality of robot walking. Thus, considering our work is mainly focused on the horizontal component of the CM's states, $w_{0}$ is set to zero and the rod motion is only translational just prior to the impact. Meanwhile, for the qualitative description consistency of $u_{f}$ and $u_{i}$, just at the end of swing phase, the inverted pendulum is rigid and only rotating around the $\mathrm{CP}$. As the result, $u_{f c a l}$ is the horizontal component of the inverted pendulum CM's tangential velocity, as shown in Fig. 6(d).

For more efficient in the calculation, let $\lambda^{\prime}=1-\lambda$ and yields:

$$
u_{f c a l}=U_{\text {rod }}\left(\lambda, v, u, x, y, L_{S}, m\right)
$$

The full expression of $U_{\text {rod }}\left(\lambda, v, u, x, y, L_{S}, m\right)$ is detailed in Appendix 2.

\subsection{The Calculation of $\lambda$}

With the sensed initial state $\left(v_{0}, u_{0}, w_{0}, x_{i}, y_{i}\right)$ and final horizontal velocity $u_{f}$ of robot's CM during a walking cycle, (24) with variable $\lambda$ can be solved by a linearization at the point $\lambda=0$ :

$$
\begin{aligned}
\lambda & =\lambda\left(v_{0}, u_{0}, x_{i}, y_{i}, L_{S}, m, u_{f}\right) \\
& =\frac{u_{f}-U_{\text {rod }}\left(0, v_{0}, u_{0}, x_{i}, y_{i}, L_{S}, m\right)}{\frac{\partial}{\partial \lambda} U_{\text {rod }}\left(\lambda, v_{0}, u_{0}, x_{i}, y_{i}, L_{S}, m\right) \mid \lambda=0}
\end{aligned}
$$




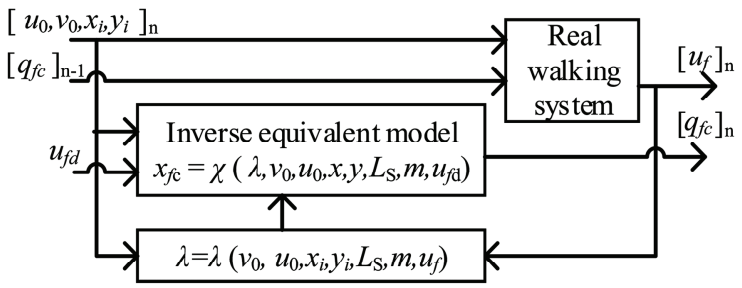

Figure 7. Structure of adaptive feedforward control.

\subsection{Controller Design}

Finally, with the calculated $\lambda$ and the desired $u_{f}$, denoted by $u_{f d}, x_{f c}$ can be solved by a linearization at the point $x=x_{i}$ :

$$
\begin{aligned}
x_{f c} & =\chi\left(\lambda, v_{0}, u_{0}, y_{i}, L_{S}, m, u_{f d}\right) \\
& =x_{f}+\frac{u_{f d}-U_{\text {rod }}\left(\lambda, v_{0}, u_{0}, x_{i}, y_{i}, L_{S}, m\right)}{\frac{\partial}{\partial x} U_{\text {rod }}\left(\lambda, v_{0}, u_{0}, x, y_{i}, L_{S}, m\right) \mid \lambda=0}
\end{aligned}
$$

Regardless of the ground compliance, with the preplanned gait, the first walking cycle must be realized, thus, in practice, by setting $u_{f d}$ equaling to $u_{f}$ of the first cycle, the whole walking process will be stabilized by suppressing the variation of walking speed. The structure of feedforward control strategy is shown in Fig. 7. With it, the development of the adaptive feedforward controller which is only based on the sensed robot walking state is completed.

\subsection{Simulation of Controlled Walking on the Com- pliant Ground}

Compared with the uncontrolled walking on a compliant ground, for a controlled walking cycle, the desired final state for the trajectories of actuated joints generation is updated with $\left[q_{f c}, \dot{q}_{f}\right]$, where $q_{f c}$ is the controlled input calculated through (25) and the method detailed in Appendix 1.

\section{Evaluation of the Control Strategy}

To validate the availability of the control strategy, the uncontrolled and controlled walking processes initialized with the preplanned gait (denoted with $I G$ 1) under the three ground conditions were compared. Then, to demonstrate the generality and adaptability of the feedforward control strategy, another two initial gaits ( $I G 2$ and $I G 3)$ and three compliant ground constructed with randomly generated damper array were considered in the numerically experiment.

In this paper, all of the robot's walking states were obtained with MATLAB software by using the functions, ODE23s and ODE15s, to solve the ordinary differential equations (5)' numerically.

\subsection{Effect of Ground Compliance on Underactu- ated Walking}

The parameters of the robot structure are shown in Table 1. The initial states of the gait preplanned under rigid robot-ground contact assumption are given in Table 2. The three ground conditions for the simulation are listed in Table 3.

Considering the robot's state is positively related to $\dot{q}_{1}$, two indicators are used for quantifying its changes through a full walking cycle:

1. $R_{S}\left(R_{S}:=\dot{q}_{1 S S P}^{-} / \dot{q}_{1 S S P}^{+}\right.$, where "-" / "+" denotes the value before/after a SSP).

2. $R_{D}\left(R_{D}:=\dot{q}_{1 D S P}^{+} / \dot{q}_{1 D S P}^{-}\right.$, where "+"/"-" denotes the value after/before a DSP)

The output is shown in Fig. 8. Meanwhile, three groups of observed values were collected when the stable states reached and shown in Table 4: (1) the number of cycles for reaching a stable state, denoted with $N_{S} ;(2)$ the robot's walking speed, denoted with ws (defined in Fig. 5); (3) the relative position of the robot's CM $\left(P_{r c}=x_{f} / L_{S}\right)$. For further analysing the effect of compliant ground on the preplanned gait, the values of the first walking cycle

Table 1

Parameters of Robot Structure

\begin{tabular}{|l|c|c|}
\hline$l(\mathrm{~m})$ & $m_{1} \sim m_{6}(\mathrm{~kg})$ & $m_{7}(\mathrm{~kg})$ \\
\hline 0.35 & 0.5 & 1.0 \\
\hline
\end{tabular}

Table 2

Initial State of $I G 1$

\begin{tabular}{|c|r|r|c|c|r|}
\hline \multirow{2}{*}{$q_{i}(\mathrm{rad})$} & $q_{1 i}$ & -0.1708 & $\dot{q}_{i}(\mathrm{rad} / \mathrm{s})$ & $\dot{q}_{1 i}$ & 1.0840 \\
\cline { 2 - 2 } & $q_{2 i}$ & 0.1500 & & $\dot{q}_{2 i}$ & 0.0169 \\
\cline { 2 - 3 } & $q_{3 i}$ & -0.3094 & & $\dot{q}_{3 i}$ & 0.1420 \\
\cline { 2 - 3 } & $q_{4 i}$ & -0.2500 & & $\dot{q}_{4 i}$ & -0.0579 \\
\hline
\end{tabular}

Table 3

Properties of Compliant Ground

\begin{tabular}{|l|l|l|}
\hline & $c(\mathrm{~N} \cdot \mathrm{s} / \mathrm{m})$ & $k(\mathrm{~N} / \mathrm{m})$ \\
\cline { 1 - 2 } Condition 1 & $0.02 \times 10^{4}$ & \multirow{2}{*}{$2 \times 10^{4}$} \\
\cline { 1 - 2 } Condition 2 & $0.30 \times 10^{4}$ & \\
\cline { 1 - 2 } Condition 2 & $0.60 \times 10^{4}$ & \\
\hline
\end{tabular}

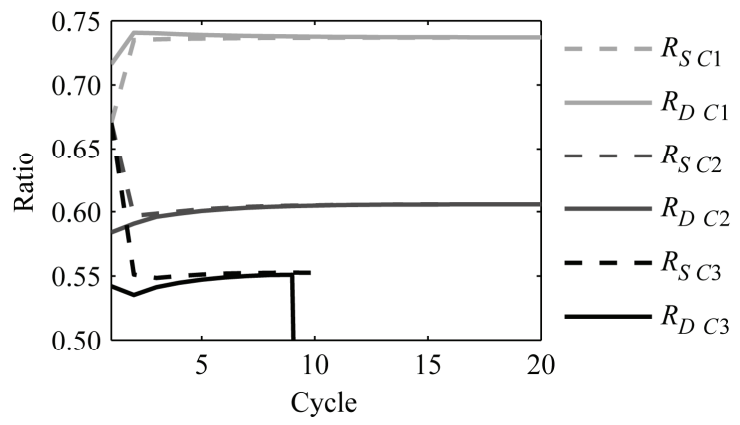

Figure 8. Convergence process of $R_{S}$ and $R_{D}$ (subscript "Ci": Condition i). 
Table 4

Walking Performance on Compliant Ground

\begin{tabular}{|l|c|c|c|c|}
\hline & First cycle & $(1)^{\mathrm{a}}$ & $(2)$ & $(3)$ \\
\hline$N_{S}$ & - & 14 & 10 & 9 \\
\hline ws $(\mathrm{m} / \mathrm{s})$ & 0.4261 & 0.4891 & 0.3382 & 0.1964 \\
\hline$P_{r c}$ & 0.4364 & 0.4367 & 0.4319 & 0.4135 \\
\hline
\end{tabular}

a $(i)$ denotes the underactuated bipedal walking process under Condition $i$.

were also collected in Table 4 . It must be noted, under Condition 3 , these values were calculated in the cycle just before the divergence occurred.

Three phenomena about the relationship between the change of $\dot{q}_{i}$ and the walking performance were concluded:

1. Under Condition $1, R_{S}$ was lower than $R_{D}$ initially and both of them converged to an identical value after the 14th cycle. Meanwhile, the walking system reached a periodic stable state with increased speed and $x_{f}$.

2. Under Condition $2, R_{S}$ was higher than $R_{D}$ initially and both of them converged to an identical value after the 10th cycle. Meanwhile, the walking system reached a periodic stable state with decreased speed and $x_{f}$.

3. Under Condition $3, R_{S}$ was higher than $R_{D}$ and both of them diverged after the ninth cycles. Meanwhile, the walking system fell.

The further analysis is detailed in [32]. Because of the diversity of initial gait and ground compliance, it is impossible to figure out all the situations when an underactuated robot is walking on a compliant ground. In spite of that, it is undoubted that the stability and the performance of the underactuated bipedal walking is actually affected by the ground compliance. It is also in accord with the result in the literature.

\subsection{Availability of the Equivalent Model Establish- ment}

To validate the equivalent model establishment available, from the second to the seventh cycle, $u_{f c a l}$ and $v_{f c a l}$, the calculated vertical velocity, were calculated through
(26) and (25) under the conditions shown in Table 3 and compared to the counterparts of the actual robot's CM velocities. The results are shown in Fig. 9.

1. In each cycle, $u_{f c a l}$, is almost identical to $u_{f}$.

2. The difference between $v_{f c a l}$ and $v_{f}$ is comparatively larger. However, both $v_{f c a l}$ and $v_{f}$ are downward and have the same varying tendency. Furthermore, with the ground damping increasing, the difference between them of each cycle is decreased.

In conclusion, despite the existing error between $v_{f c a l}$ and $v_{f}$, as $u_{f}$ is the only concerned in the SISO control and has been equated with $u_{f c a l}$ perfectly, this method through establishing a rod-ground equivalent model to simplify the high dimensional robot-ground model is available.

\subsection{Effectiveness of the Adaptive Feedforward Con- troller}

To demonstrate the performance of the adaptive feedforward controller, the controller only worked once at the end of the second walking cycle. The schedule of the controlled walking process is shown in Fig. 10. Under the three conditions listed in Table 3, the comparisons between the uncontrolled $u_{f}$, denoted by $u_{f u c}$, and controlled $u_{f}$, denoted by $u_{f c}$, are depicted in Fig. 11. Meanwhile, the steady-state errors were also collected and shown in Table 5. Let $u_{f 1}$ denote the actual $u_{f}$ of the first cycle.

1. The control strategy is effective. Under the three conditions, compared with $u_{f u c}$, the variations of $u_{f c}$ were all suppressed significantly after the third cycle. And then, each $u_{f c}$ was converged to $u_{f 1}$ rapidly. After the 18th cycle, all $u_{f c}$ reached the periodic stable states, as shown in Fig. 11.

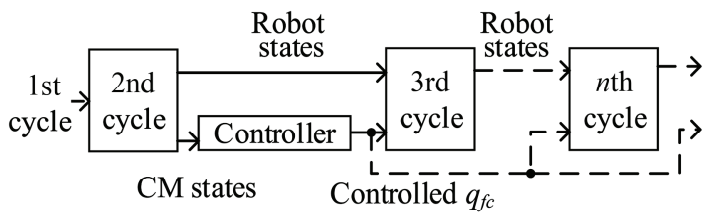

Figure 10. Schedule of the controlled walking process.

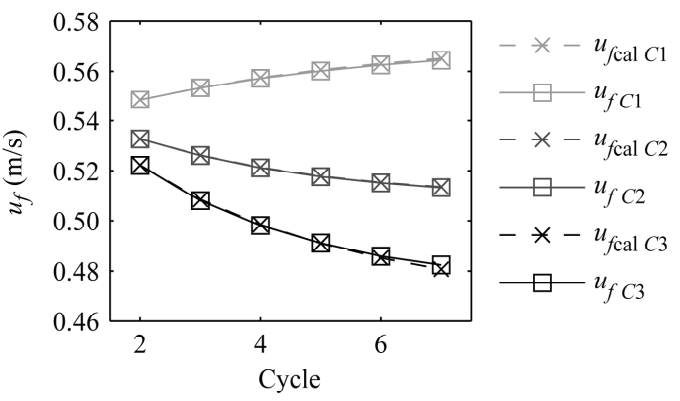

(a)

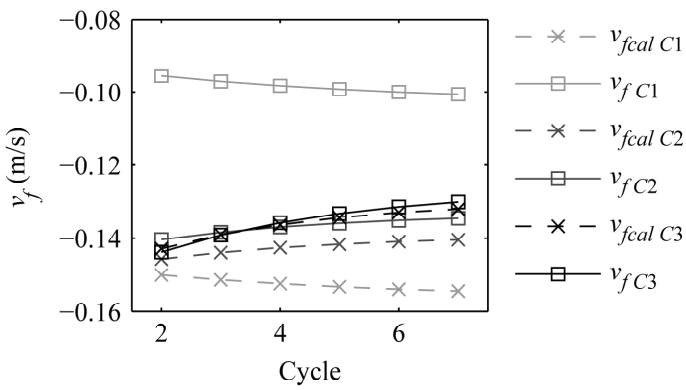

(b)

Figure 9. Comparison between equivalent rod-ground model and original robot-ground model (subscript " $C i$ ": Condition $i$ ). (a) Comparison of the horizontal velocities and (b) comparison of the vertical velocities. 
Table 5

Comparison of Relative Error Between Controlled and Uncontrolled $u_{f}$

\begin{tabular}{|l|c|c|c|}
\hline & $u_{f 1}(\mathrm{~m} / \mathrm{s})$ & $E_{r}$ for $u_{f u c}$ & $E_{r}$ for $u_{f c}$ \\
\hline Condition 1 & 0.5419 & $5.43 \%$ & $0.09 \%$ \\
\hline Condition 2 & 0.5405 & $5.82 \%$ & $0.72 \%$ \\
\hline Condition 2 & 0.5374 & $11.11 \%($ ninth) & $0.87 \%$ \\
\hline
\end{tabular}

Table 6

$P_{r c}$ of Controlled Walking Process

\begin{tabular}{|c|c|c|c|}
\hline & $(1)^{\mathrm{b}}$ & $(2)$ & $(3)$ \\
\hline$P_{r c}$ & 0.4282 & 0.4557 & 0.4674 \\
\hline
\end{tabular}

b (i) denotes the underactuated bipedal walking process under Condition $i$.

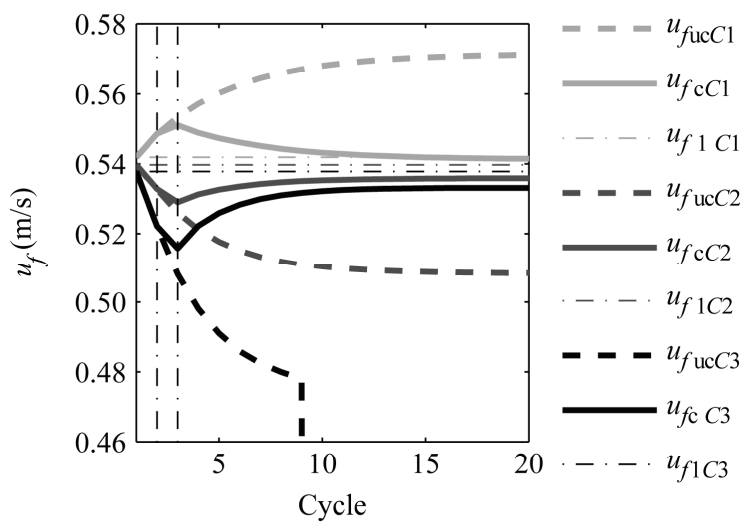

Figure 11. Performance of the adaptive feedforward controller in tracking $u_{f d}$ (subscript "Ci": Condition i.)

2. The steady-state performance of the controlled walking is acceptable. Looking into the comparison of steady-state errors shown in Table 5, under the three conditions, the relative errors $\left(E_{r}:=\left(u_{f}-u_{f 1}\right) / u_{f 1}\right)$ of $u_{f c}$ is smaller than $u_{f u c}$ significantly. This result also validate the availability of the equivalent model establishment.

3. The variation of controlled input is in accord with the inspiration of the controller design. For example, if the acceleration was needed, $x_{f c}$ was increased, such as the situations when the robot walked under Condition 2 and Condition 3, as shown in Table 6 .

\subsection{Availability of the Stabilization Control Strat- egy}

To further evaluate the effectiveness of the adaptive feedforward control strategy in stabilizing the walking on compliant ground, the rates of stable convergence for controlled walking processes under the three conditions listed in Table 3 are depicted in Fig. 12, meanwhile, the comparison between the controlled and uncontrolled walking speeds are shown in Fig. 13.

According to Figs. 12 and 13:

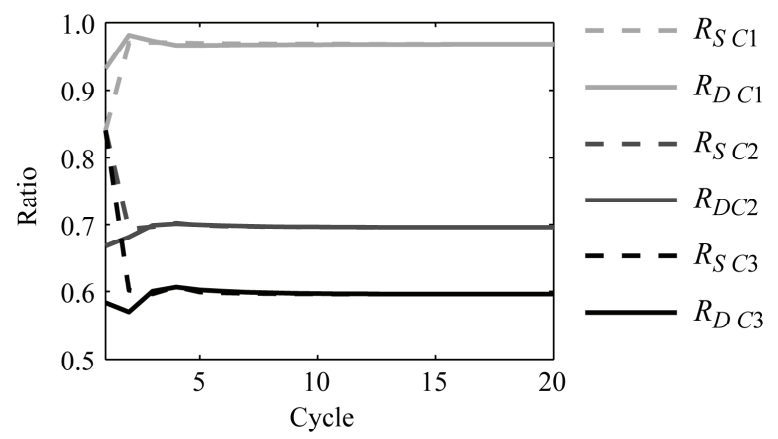

Figure 12. Convergence process of $R_{D}$ and $R_{S}$ with the adaptive feedforward controller (subscript " $\mathrm{C} i$ ": Condition i.)

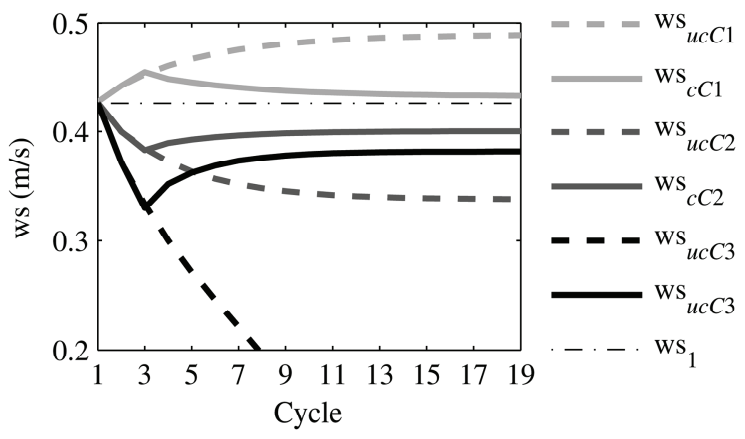

Figure 13. Comparison between controlled and uncontrolled walking speed (ws: walking speed; subscript "uc": uncontrolled; subscript "c": controlled; subscript " $\mathrm{C} i$ ": Condition $i$ ).

1. The proposed feedforward control strategy is able to stabilize the underactuated bipedal walking on the compliant ground. With the controller, all walking processes were stabilized. In particular, compared with the uncontrolled walking under Condition 3, the controlled walking speed just decreased before the third cycle, but increased dramatically after that, and achieved a periodic stable state in the 16 th cycle.

2. The control strategy improves both the rate of convergence and the performance of inherently stable walking system. According to the convergence processes of $R_{S}$ and $R_{D}$ shown in Fig. 12, all coincidences between $R_{S}$ and $R_{D}$ for the first time occurred in the third cycle. Compared with the uncontrolled convergences shown in Fig. 5, the rates of stable convergence for controlled walking system were increased significantly. Meanwhile, with the controller, the robot's walking performance were improved as well. Both under Condition 1 and Condition 2, the controlled walking speeds were much closer to the actual speed of the first cycle than the uncontrolled, as shown in Fig. 13. 

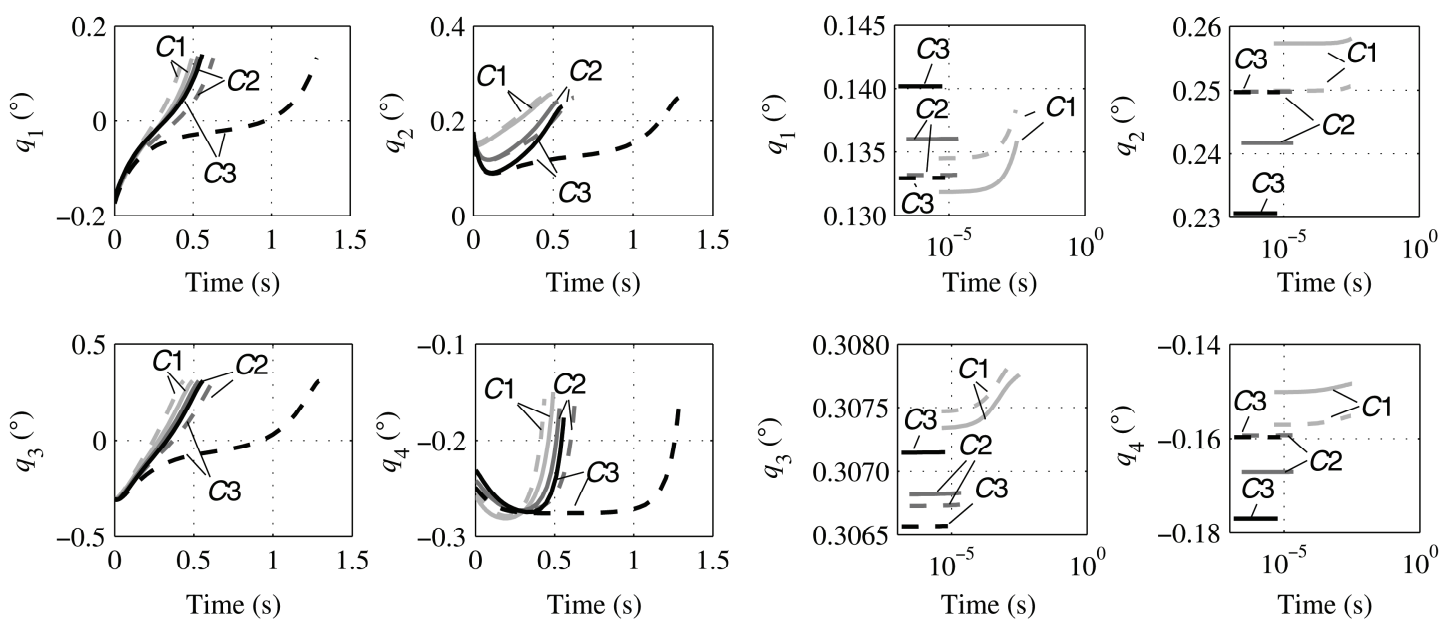

(a)

(b)

Figure 14. Comparison of joint trajectories between a controlled and uncontrolled walking cycle. (a) Joint trajectories during SSP and (b) Joint trajectories during DSP. (Dash line: the uncontrolled walking; solid line: the controlled walking; $C i$ : walking under Condition $i$. Particularly, except the black dashed lines collected during the ninth cycle, these trajectories are all collected during the 21st cycle.)

To further analyse the effect of the adaptive feedforward control strategy on the walking under the compliant ground environment, the robot's joints trajectories during a full walking cycle when the stable state reached are depicted under the three conditions, as shown in Fig. 14.

According to Fig. 14:

1. Without the controller, only under the effect of ground compliant, with the ground damping increasing, despite the total variation of each joint position through a SSP being almost consistent, the duration of the SSP is increased and the duration of the DSP decreased dramatically.

2. With the controller, the difference between the durations of SSP under the three conditions is reduced significantly, but the durations of DSP were almost invariant.

3. At the end of the SSP, under Condition 1, the controlled $q_{2}$ is larger than the uncontrolled but the controlled $q_{4}$ smaller than the uncontrolled simultaneously, on the contrary, under Condition 2 and Condition 3, the controlled $q_{2}$ is smaller than the uncontrolled but the controlled $q_{4}$ larger than the uncontrolled. It is in accord with the results of comparisons between $x_{f}$ and $x_{f c}$ shown in Tables 4 and 6 and the mapping relation detailed in Appendix 1.

\subsection{Adaptability of the Adaptive Feedforward Con- trol Strategy}

To demonstrate the generality of the control strategy, two more initial gaits, shown in Tables 7 and 8 , were used in the simulation experiments. Meanwhile, to demonstrate the adaptability of the feedforward control strategy, the compliant ground with stochastic damping parameters was also considered:

1. The coefficients of damping were generated randomly in MATLAB with RANDI function. Three
Table 7

Initial State of $I G 2$

\begin{tabular}{|c|c|c|c|c|c|}
\hline \multirow[t]{4}{*}{$q_{i}(\mathrm{rad})$} & $q_{1 i}$ & -0.1660 & \multirow[t]{4}{*}{$\dot{q}_{i}(\mathrm{rad} / \mathrm{s})$} & $\dot{q}_{1 i}$ & 1.1651 \\
\hline & $q_{2 i}$ & 0.1650 & & $\dot{q}_{2 i}$ & -0.0269 \\
\hline & $q_{3 i}$ & -0.3094 & & $\dot{q}_{3 i}$ & 0.1481 \\
\hline & $q_{4 i}$ & -0.2350 & & $\dot{q}_{4 i}$ & -0.1293 \\
\hline
\end{tabular}

Table 8

Initial State of $I G 3$

\begin{tabular}{|c|r|r|r|r|r|}
\hline$q_{i}(\mathrm{rad})$ & $q_{1 i}$ & -0.1546 & $\dot{q}_{i}(\mathrm{rad} / \mathrm{s})$ & $\dot{q}_{1 i}$ & 1.3284 \\
\cline { 2 - 2 } & $q_{2 i}$ & 0.2001 & & $\dot{q}_{2 i}$ & 0.2686 \\
\cline { 2 - 2 } & $q_{3 i}$ & -0.3093 & & $\dot{q}_{3 i}$ & 0.1456 \\
\cline { 1 - 2 } & $q_{4 i}$ & -0.2003 & & $\dot{q}_{4 i}$ & -0.3135 \\
\hline
\end{tabular}

array-of-the-coefficient-of-damping (ACD) are shown in Table 9. To cover the conditions shown in Table 3, ACD 1 and ACD 2 were both ranging from $0.01 \times 10^{4} \mathrm{~N} \cdot \mathrm{s} / \mathrm{m}$ to $1 \times 10^{4} \mathrm{~N} \cdot \mathrm{s} / \mathrm{m}$, and for further exploring the performance of the adaptive feedforward control strategy, ACD 3 was ranging from $0.01 \times 10^{4} \mathrm{~N} \cdot \mathrm{s} / \mathrm{m}$ to $3 \times 10^{4} \mathrm{~N} \cdot \mathrm{s} / \mathrm{m}$.

2. The coefficients of damping changed once every four walking cycles. It means, in every four cycles, the robot would go through two kinds of DSP: (1) the coefficients of damping beneath the both legs were different, shown as the situation (a) in Fig. 15; (2) the coefficients of damping beneath the both legs were identical, shown as the situation (b) in Fig. 15.

3. Accordingly, to cope with the non-homogeneous compliant ground, the controller worked once at the end of every two walking cycles. Define the first cycle of the 
Table 9

Arrays of Randomly Generated Coefficients of Damping $\left(\times 10^{4} \mathrm{~N} \cdot \mathrm{s} / \mathrm{m}\right)$

\begin{tabular}{|l|c|c|c|c|c|c|c|}
\hline & ACD1 & ACD2 & ACD3 & & ACD1 & ACD2 & ACD3 \\
\hline$c_{1}$ & 0.83 & 0.52 & 1.69 & $c_{6}$ & 0.40 & 0.41 & 1.39 \\
\hline$c_{2}$ & 0.43 & 0.89 & 2.65 & $c_{7}$ & 0.81 & 0.75 & 2.95 \\
\hline$c_{3}$ & 0.89 & 0.59 & 2.01 & $c_{8}$ & 0.76 & 0.83 & 0.47 \\
\hline$c_{4}$ & 0.40 & 0.16 & 0.58 & $c_{9}$ & 0.38 & 0.79 & 2.57 \\
\hline$c_{5}$ & 0.77 & 0.20 & 1.11 & $c_{10}$ & 0.22 & 0.32 & 1.94 \\
\hline
\end{tabular}

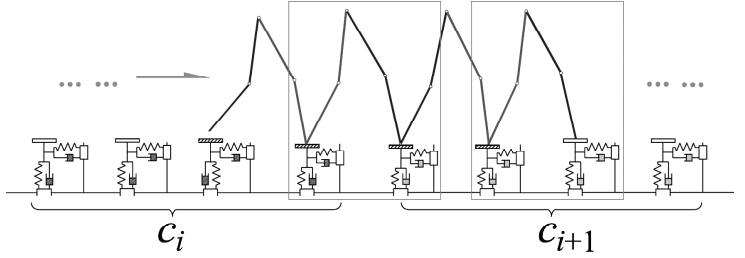

(a)

(b)

Figure 15. Walking on compliant ground with stochastic damping parameters $\left(c_{i}\right.$ and $c_{i+1}$ are two different coefficients of damping).

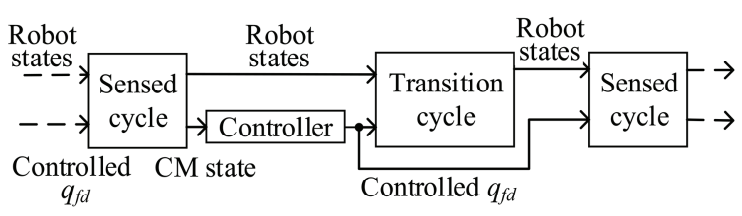

Figure 16. Schedule of control strategy.

two is the sensed cycle and the other is the transition cycle. The schedule of the controlled walking process is described as Fig. 16.

The comparisons between controlled and uncontrolled walking speeds are shown in Fig. 17. The final states of controlled and uncontrolled walking processes are detailed in Table 10, meanwhile, the mean speed of each whole walking process is calculated and shown in Table 11.

According to Fig. 17, Tables 10 and 11:

1. All controlled underactuated bipedal walking on the compliant ground were stabilized. For all instable gaits without controller (e.g., initialized with $I G 1$ and $I G$ 2 ), all of them had fallen down when the ground was increasingly damped or a relatively large damping had been lasting for some cycles. Based on these phenomena, the instability shall be attributed to lacking of stall control. In comparison with this, regardless of the ground conditions, the variation of the controlled walking speed had been effectively controlled.

2. Compared with the uncontrolled walking, the controlled was more efficiently. Specifically speaking, looking into the walking processes initialized with $I G$ 3 , regardless of the ground conditions, despite all the processes being inherently stable without controller,

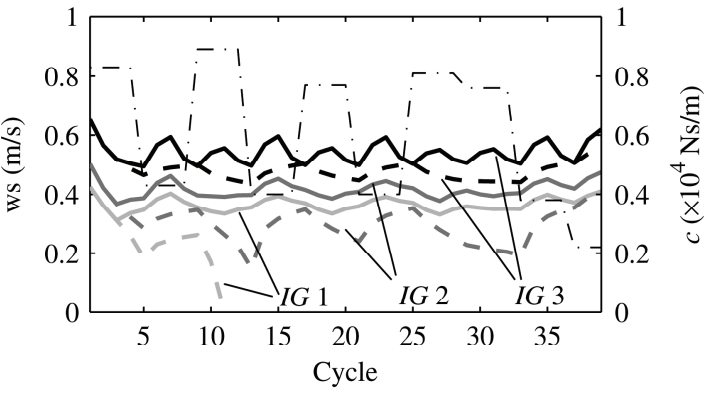

(a)

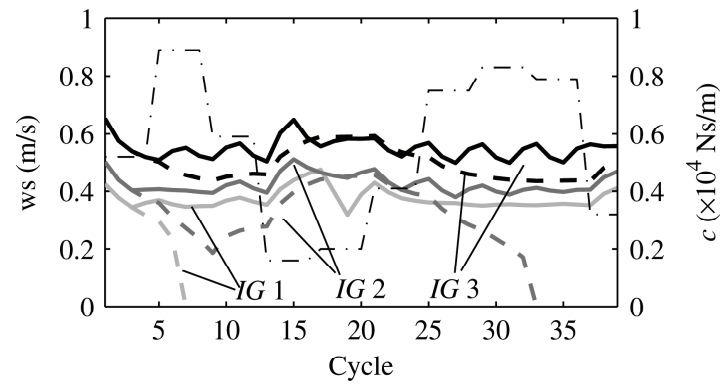

(b)

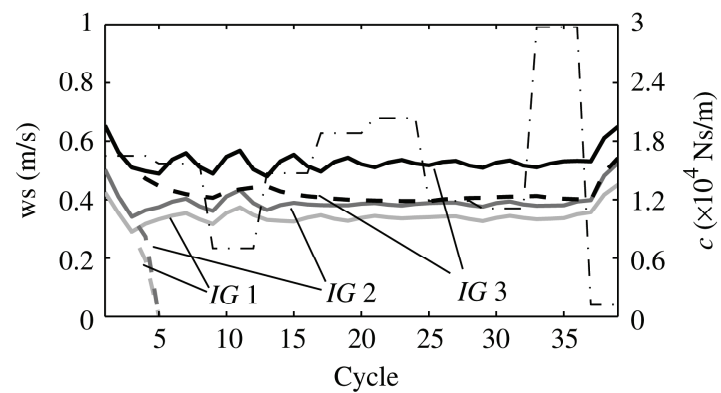

(c)

Figure 17. Walking on the stochastically damped compliant ground. (a) Walking on the compliant ground constructed with ACD 1; (b) Walking on the compliant ground constructed with ACD 2; and (c) Walking on the compliant ground constructed with ACD 3. (ws: walking speed; dash line: uncontrolled ws; solid line: controlled ws; $I G i$ : walking initialized with $I G i$; black dash-dot line: damping parameters.)

within the same distance passed through, the controlled walking system took less time to complete it than the uncontrolled significantly, as shown in Fig. 18, especially when the ground damping was relatively 
Table 10

The Final State of Walking Process

\begin{tabular}{|l|c|c|c|}
\hline & ACD 1 & ACD 2 & ACD 3 \\
\hline$I G 1 \& \mathrm{c}^{\mathrm{a}}$ & $\mathrm{S}^{\mathrm{c}}$ & $\mathrm{S}$ & $\mathrm{S}$ \\
\hline$I G 1 \& \mathrm{uc}^{\mathrm{b}}$ & $\mathrm{F}^{\mathrm{d}}(9 \mathrm{th})$ & $\mathrm{F}(5 \mathrm{th})$ & $\mathrm{F}(4 \mathrm{th})$ \\
\hline$I G 2 \& \mathrm{c}$ & $\mathrm{S}$ & $\mathrm{S}$ & $\mathrm{S}$ \\
\hline$I G 2 \& \mathrm{uc}$ & $\mathrm{S}$ & $\mathrm{F}(31 \mathrm{st})$ & $\mathrm{F}(4 \mathrm{th})$ \\
\hline$I G 3 \& \mathrm{c}$ & $\mathrm{S}$ & $\mathrm{S}$ & $\mathrm{S}$ \\
\hline$I G 3 \& \mathrm{uc}$ & $\mathrm{S}$ & $\mathrm{S}$ & $\mathrm{S}$ \\
\hline
\end{tabular}

a "c" denotes "controlled"

b "uc" denotes "uncontrolled"

c "S" denotes "Stable"

d "F" denotes "Fallen"

e(i) denotes the process falls after the $i$ th cycle

Table 11

The Mean Speed of the Whole Walking Process $(\mathrm{m} / \mathrm{s})$

\begin{tabular}{|l|c|c|c|}
\hline & ACD 1 & ACD 2 & ACD 3 \\
\hline$I G 1 \& \mathrm{c}$ & 0.3652 & 0.3751 & 0.3447 \\
\hline$I G 1 \& \mathrm{uc}$ & $0.2818^{\mathrm{a}}$ & 0.3522 & 0.3561 \\
\hline$I G 2 \& \mathrm{c}$ & 0.4169 & 0.4271 & 0.3943 \\
\hline$I G 2 \& \mathrm{uc}$ & 0.4036 & 0.3490 & 0.4181 \\
\hline$I G 3 \& \mathrm{c}$ & 0.5434 & 0.5513 & 0.5326 \\
\hline$I G 3 \& \mathrm{uc}$ & 0.4844 & 0.5007 & 0.4311 \\
\hline
\end{tabular}

a. The value for the fallen process was the mean speed before the walking fell.

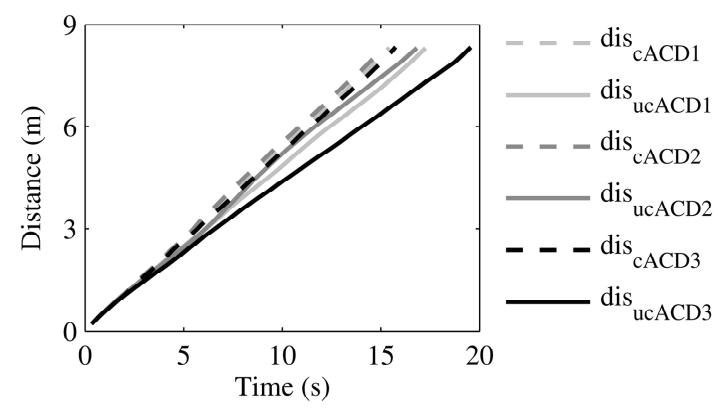

Figure 18. Performance improvement of walking process initialized with $I G 3$ (dis: distance; subscript "c": controlled; subscript "uc": uncontrolled).

high, e.g., when the robot walked on the compliant ground constructed with ACD 3, the time of controlled walking process was faster than the uncontrolled by approximate $3.8 \mathrm{~s}$. It will make the bipedal robot work more efficient in the daily routine work.

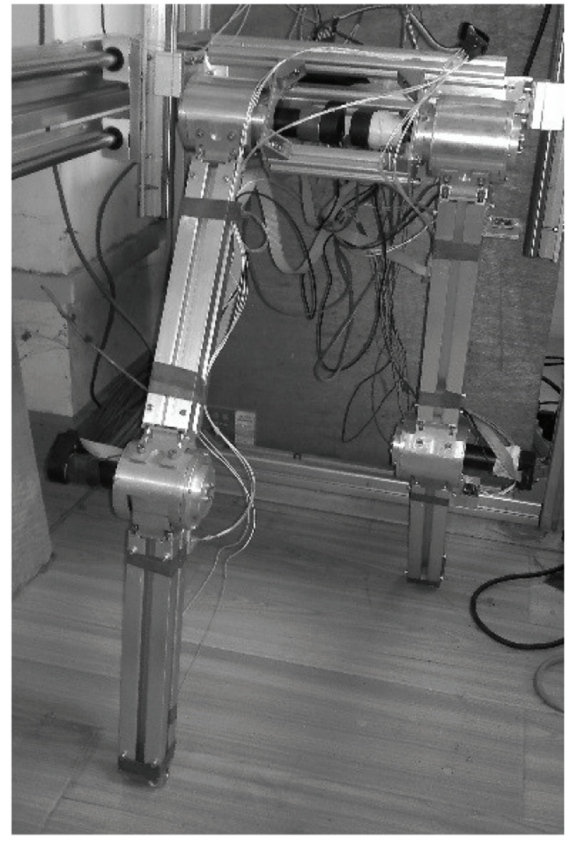

Figure 19. Prototype robot "UBbot".

\section{Conclusion and Future Work}

This paper proposes an adaptive feedforward control strategy to control the robot's speed by regulating the CM motion and further stabilize the underactuated bipedal walking on the compliant ground.

1. This control strategy achieves good adaptability for stabilizing the underactuated bipedal walking on compliant ground. As only the effect of the ground beneath the robot on the walking performance is considered in the controlled input calculation, this method is essentially adaptable to the ground compliance varying and other bipedal walking system.

2. The control strategy is low cost in computation source for real-time motion control. As the walking system is modelled as a polynomial with definite number of degrees and the controlled input is derived through a simple inverse operation on it, the control algorithm is fast and stable. As the result, this control strategy can be easily implemented in the real control system and has a good real-time performance.

3. The control strategy has broad application prospects. As the control strategy is originated from the human's gait, it can be easily integrated into a more advanced bionic control system to realize a multi-task walking mission in a human-like manner.

Our future work is focus on two points: (1) try to realize the control strategy on our prototype robot shown in Fig. 19; (2) try to find a more efficient control strategy to eliminate the steady error between the controlled walking speed and the desired walking speed.

\section{Acknowledgement}

This research is supported by National Natural Science Foundation of China (NSFC, Grant No.51175383) 


\section{Appendix 1}

Suppose a designed robot's configuration at the end of a SSP is denoted as $q_{f}=\left[q_{1}, q_{2}, q_{3}, q_{4}\right]_{f}^{\prime}$. Considering the mapping relation from $x_{f c}$ to $q_{f c}=\left[q_{1}, q_{2}, q_{3}, q_{4}\right]_{f c}^{\prime}$ is a multi-solution problem, two more constraints are introduced:

1. $L_{S}$ is a constant;

2. $q_{2 f c}=q_{2 f}+\Delta q$ and $q_{4 f c}=q_{4 f}+\Delta q$.

Second, according to the relationship between $x_{f c}$ and $\Delta q$, yield:

$$
x_{f c}=\frac{1}{16}[11 l \sin (\alpha)+15 l \sin (\beta)-l \sin (\gamma)-5 l \sin (\delta)]
$$

with

$$
\begin{array}{ll}
\alpha=q_{1 f c}-\frac{q_{2 f c}}{2} & \beta=q_{1 f c}+\frac{q_{2 f c}}{2} \\
\gamma=q_{1 f c}-q_{3 f c}-\frac{q_{4 f c}}{2} & \delta=q_{1 f c}-q_{3 f c}+\frac{q_{4 f c}}{2} \\
l_{1}=\sqrt{2 l^{2}+2 l^{2} \cos \left(q_{2 f}+\Delta q\right)} & l_{2}=\sqrt{2 l^{2}+2 l^{2} \cos \left(q_{4 f}+\Delta q\right)} \\
q_{3 f c}=\arccos \left(\frac{l_{1}^{2}+l_{2}^{2}-L_{S}^{2}}{2 l_{1} l_{2}}\right) & q_{1 f c}=\arccos \left(\frac{l_{2} \sin \left(q_{3 f c}\right)}{L_{S}}\right)
\end{array}
$$

Then the mapping relation from $x_{f}$ to $\Delta q$ is derived through a first-order linearization:

$$
\Delta q=\frac{x_{f c}-x_{f}}{\partial x_{f c} / \partial \Delta q}
$$

\section{Appendix 2}

$$
\begin{aligned}
& U_{\text {rod }}\left(\lambda, v, u, x, y, L_{S}, m\right) \\
& =\frac{\sqrt{3} y}{2 \sqrt{\left(L_{S}-x\right)^{2}+y^{2}}} \sqrt{a+b+c+d}
\end{aligned}
$$

where

$$
\begin{aligned}
a & =-\frac{g L_{S}\left(L_{S}-2 x\right)(-1+\lambda)}{y} \\
b & =\left(u_{0}-\frac{m_{\rho} u_{0} \lambda}{m}\right)^{2} \\
c & =\frac{\left[2 \rho^{2} m\left(m-c t_{1}\right) v_{0}+c m_{\rho} t_{1} u_{0} x y \lambda\right]^{2}}{4 \rho^{4} m^{4}} \\
d & =\frac{\left[2 \rho^{2} m m_{\rho} u_{0} y \lambda+c t_{1} x\left(2 \rho^{2} m v_{0}-m_{\rho} u_{0} x y \lambda\right)\right]^{2}}{4 \rho^{6} m^{4}} \\
\rho & =\sqrt{\left(x^{2}+y^{2}\right) / 3} \\
t_{1} & =\frac{\rho^{2} m \lambda}{c\left(\rho^{2}+y_{i}^{2}\right)} \\
m_{\rho} & =\rho^{2} m /\left(\rho^{2}+y^{2}\right)
\end{aligned}
$$

\section{References}

[1] T. McGeer, Passive dynamic walking, International Journal of Robotics Research, 9(2), 1990, 62-82.

[2] J.W. Grizzle, C. Chevallereau, R.W. Sinnet, and A.D. Ames, Models, feedback control, and open problems of 3D bipedal robotic walking, Automatica, 50(8), 2014, 1955-1988.
[3] S. Collins, A. Ruina, R. Tedrake, and M. Wisse, Efficient bipedal robots based on passive-dynamic walkers, Science, 307(5712), 2005, 1082-1085.

[4] C. Chevallereau, A. Gabriel, Y. Aoustin, F. Plestan, E. Westervelt, C.C. De Wit, and J. Grizzle, Rabbit: A testbed for advanced control theory, IEEE Control Systems Magazine, 23(5), 2003, 57-79.

[5] A. Ramezani and J.W. Grizzle, ATRIAS 2.0, a new 3-D bipedal roboticwalker and runner, Proc. International Conference on Climbing and Walking Robots and the Support Technologies for Mobile Machines, 2012, 467-474.

[6] H. Dai, A. Valenzuela, and R. Tedrake, Whole-body motion planning with centroidal dynamics and full kinematics, Proc. IEEE-RAS International Conference on Humanoid Robots, 2014, 295-302.

[7] J. Grizzle, J. Hurst, B. Morris, H.-W. Park, and K. Sreenath, MABEL, a new robotic bipedal walker and runner, Proc. American Control Conference, 2009, 2030-2036.

[8] S.N. Yadukumar, M. Pasupuleti, and A.D. Ames, Humaninspired underactuated bipedal robotic walking with AMBER on flat-ground, up-slope and uneven terrain, Proc. IEEE/RSJ International Conf. Intelligent Robots and Systems, 2012, 2478-2483.

[9] B. Tondu and N. Bardou, A new interpretation of mori's uncanny valley for future humanoid robots, International Journal of Robotics 83 Automation, 26(3), 2011, 1.

[10] W. Stronge, R. James, and B. Ravani, Oblique impact with friction and tangential compliance, Philosophical Transactions of the Royal Society of London A: Mathematical, Physical and Engineering Sciences, 359(1789), 2001, 2447-2465.

[11] Y.-T. Wang, V. Kumar, and J. Abel, Dynamics of rigid bodies undergoing multiple frictional contacts, Proc. IEEE Int. Conf. Robotics and Automation, 1992, 2764-2769.

[12] F. Plestan, J.W. Grizzle, E.R. Westervelt, and G. Abba, Stable walking of a 7-dof biped robot, IEEE Transactions on Robotics E Automation, 19(4), 2003, 653-668.

[13] E.R. Westervelt, J.W. Grizzle, C. Chevallereau, J.H. Choi, and B. Morris, Feedback control of dynamic bipedal robot locomotion (Boca Raton: CRC Press, 2007).

[14] K. Sreenath, H.W. Park, I. Poulakakis, and J.W. Grizzle, A compliant hybrid zero dynamics controller for stable, efficient and fast bipedal walking on MABEL, International Journal of Robotics Research, 30(9), 2011, 1170-1193.

[15] K. Byl and R. Tedrake, Approximate optimal control of the compass gait on rough terrain, Proc. IEEE International Conf. Robotics and Automation, 2008, 1258-1263.

[16] K. Byl and R. Tedrake, Metastable walking machines, International Journal of Robotics Research, 28(8), 2009, 1040-1064.

[17] I.R. Manchester, U. Mettin, F. Iida, and R. Tedrake, Stable dynamic walking over uneven terrain, International Journal of Robotics Research, 70(2011), 2011, 265-279.

[18] I.R. Manchester, M.M. Tobenkin, M. Levashov and R. Tedrake, Regions of attraction for hybrid limit cycles of walking robots, In Proc. IFAC World Congress, 2011, 5801-5806.

[19] Dai, Hongkai, and R. Tedrake, Optimizing robust limit cycles for legged locomotion on unknown terrain, Proc. 51st Annu. IEEE Conf. Decision and Control, 2012, 1207-1213.

[20] H. Dai and R. Tedrake, $\mathrm{L}_{2}$-gain optimization for robust bipedal walking on unknown terrain, Proc. IEEE International Conf. Robotics and Automation, 2013, 3116-3123.

[21] Y. Hurmuzlu and D.B. Marghitu, Rigid body collisions of planar kinematic chain with multiple contact points, International Journal of Robotics Research, 13(1), 1994, 82-92.

[22] T. Yang, E.R. Westervelt, A. Serrani, and J.P. Schmiedeler, A framework for the control of stable aperiodic walking in underactuated planar bipeds, Autonomous Robots, 27(3), 2009, $277-290$.

[23] J. Schröder-Schetelig, P. Manoonpong, and F. Wörgötter, Using efference copy and a forward internal model for adaptive biped walking, Autonomous Robots, 29(3-4), 2010, 357-366.

[24] T. Geng, Online regulation of the walking speed of a planar limit cycle walker via model predictive control, IEEE Transactions on Industrial Electronics, 61(5), 2014, 2326-2333.

[25] D.G.E. Hobbelen and M. Wisse, Controlling the walking speed in limit cycle walking, International Journal of Robotics Research, 27(9), 2008, 989-1005. 
[26] D.W. Marhefka and D.E. Orin, Simulation of contact using a nonlinear damping model, Proc. IEEE International Conf. Robotics and Automation, 1996, 1662-1668.

[27] P.S. Freeman and D.E. Orin, Efficient dynamic simulation of a quadruped using a decoupled tree-structure approach, International Journal of Robotics Research, 10(6), 1991, 619-627.

[28] D.E. Stewart and J.C. Trinkle, An implicit time-stepping scheme for rigid body dynamics with inelastic collisions and coulomb friction, International Journal for Numerical Methods in Engineering, 39(15), 1996, 2673-2691.

[29] W.J. Stronge, Rigid body collisions with friction, Proc. the Royal Society of London A: Mathematical, Physical and Engineering Science, 431(1881), 2000, 169-181.

[30] C. Chevallereau, J.W. Grizzle, and C.-L. Shih, Asymptotically stable walking of a five-link underactuated 3-D bipedal robot, IEEE Transactions on robotics, 25(1), 2009, 37-50.

[31] J.W. Grizzle, G. Abba, and F. Plestan, Asymptotically stable walking for biped robots: Analysis via systems with impulse effects, IEEE Transactions on Automatic Control, 446(1), 2001, 51-64.

[32] Y. Wang, J. Ding, and X. Xiao, Periodic stability for 2-D biped dynamic walking on compliant ground, International Conf. Intelligent Robotics and Applications, 2015, 369-380.

\section{Biographies}

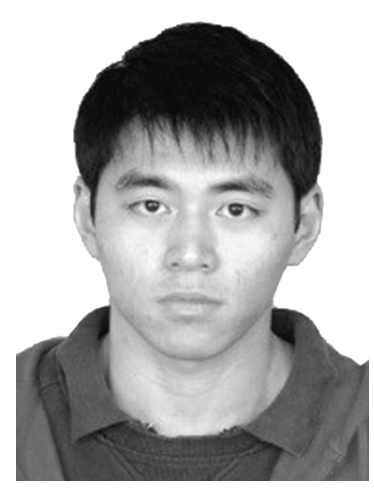

Yang Wang obtained his B.S. degree in Mechanical Design, Manufactory and Automation from Hubei University of Technology, Wuhan, in 2009. Currently, he is pursuing a Ph.D. degree in Mechatronics from Wuhan University. His research interests include dynamics, planning, and control of biped robot walking in flexible environment, and design $\&$ control of artificial bionic joint.

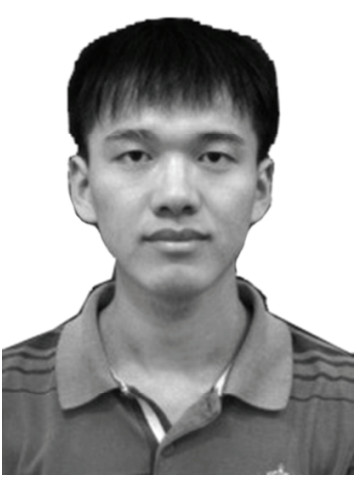

Jiatao Ding received his B.S. degree in Mechanical Engineering from Wuhan University, Wuhan, in 2010. Currently, he is pursuing a M.S. degree in Mechatronics from Wuhan University. His research interests include nonlinear dynamics, gait planning and walking control of biped robot in uncertain environment, artificial intelligence, artificial life simulation, mathematical foundations of

robotics.

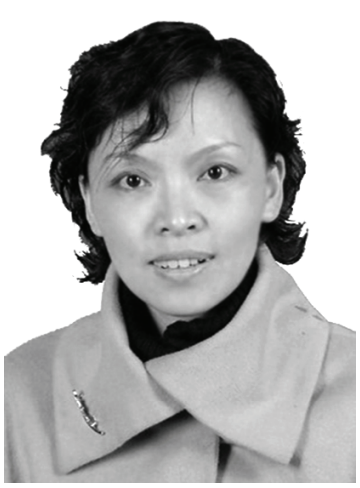

Xiaohui Xiao received her B.S. and M.S. degrees in Mechanical Engineering from Wuhan University, Wuhan, China, in 1991 and 1998, respectively, and her $\mathrm{Ph}$.D. degree in mechanical engineering from Huazhong University of Science and Technology, Wuhan, China, in 2005. She joined the Wuhan University, Wuhan, China, in 1998, where she is currently a full professor with the Mechanical Engineering Department, School of Power and Mechanical Engineering. She has published over 30 papers in the areas of mobile robots, dynamics and control, sensors and signal procession. Her current research interests include mobile robotics, high-precision positioning control, and signal processing. 